\title{
A Nonlinear Model-Based Control Method for Magnetostrictive Actuators
}

\author{
Ralph C. Smith \\ Department of Mathematics \\ Iowa State University \\ Ames, IA 50011 \\ rsmith@iastate.edu
}

\begin{abstract}
A nonlinear model-based control method for magnetostrictive actuators is presented in this paper. Such actuators utilize the realignment of magnetic moments in response to applied magnetic fields to generate strains in the material. Strains and forces generated in this manner are significantly larger than those produced by many other smart materials but also exhibit significant nonlinearities and hysteresis. To utilize the full potential of these materials in control transducers, these inherent nonlinearities and hysteresis must be accurately characterized and incorporated in the control law. An energy-based model is employed to characterize the hysteresis in a manner amenable to structural applications. Nonlinear optimal control theory is then used to determine appropriate inputs to the system. The effectiveness of this nonlinear model-based control method is demonstrated through a numerical example.
\end{abstract}

\section{Introduction}

Recent advances in the construction of magnetostrictive materials have led to the advent of actuators which have great potential in many structural applications. These actuators utilize the property that strains and forces sufficiently large to drive systems comprised of thick structures and heavy components are generated in response to applied magnetic fields. For many applications, the magnitude of the generated strains and forces makes the magnetostrictive transducers advantageous over other smart material transducers such as piezoceramics and electrostrictives. The difficulty associated with magnetostrictive actuators, however, lies in the hysteresis and nonlinearities inherent to the materials. In this paper, an energy-based model based upon domain wall interactions is used to characterize the dynamics of a magnetostrictive actuator coupled to a thin structure. This model is then discretized to obtain a finite dimensional ODE system with nonlinear control inputs. Finally, optimal control theory is used to derive a control law which utilizes the inherent nonlinearities and hysteresis.

To illustrate certain issues which must be addressed when developing a model-based control method, we consider a typical magnetostrictive transducer as depicted in Figure 1. As detailed in [3], the primary components of the transducer include a magnetostrictive rod, a wound wire solenoid, and a cylindrical permanent magnet. The sensor/actuator capabilities of the magnetostrictive material are provided by magnetic moments which rotate in the presense of an applied magnetic field. As depicted in Figure 2a, the moments are primarily oriented perpendicular to the longitudinal rod axis in the absence of an applied field. Prestressing the rod with the spring washer serves to increase the number of moments perpendicular to the axis (see Figure $2 \mathrm{~b}$ ). When a field is applied in the direction of the rod axis, moments align in the sense depicted in Figure $2 \mathrm{c}$ and significant strains and forces are generated. The driving field $H(t)$ is generated through a timedependent current $\mathcal{I}(t)$ applied to the solenoid along with the field $H_{0}$ generated by the permanent magnet. To model the transducer for structural applications, it is necessary to characterize the relationship between the current $\mathcal{I}$ applied to the solenoid, the resulting field $H$, the associated magnetization $M$, and finally the generated strains $e$. As noted in Figure 3, the relationships are highly nonlinear and exhibit significant hysteresis.

A domain wall model characterizing the hysteresis and material nonlinearities is outlined in Section 2 and incorporated in an Euler-Bernoulli thin beam model in Section 3. This illustrates the modeling of the fully coupled transducer dynamics in a typical structural application. A spline-based Galerkin method is employed in Section 4 to obtain an approximating vector ODE system. The application of optimal control theory to obtain an open loop nonlinear control input is outlined in Section 5 and illustrated through a numerical example. This example demonstrates the effectiveness of the control law and the capabilities of the transducers when nonlinear model-based control methods are employed. 


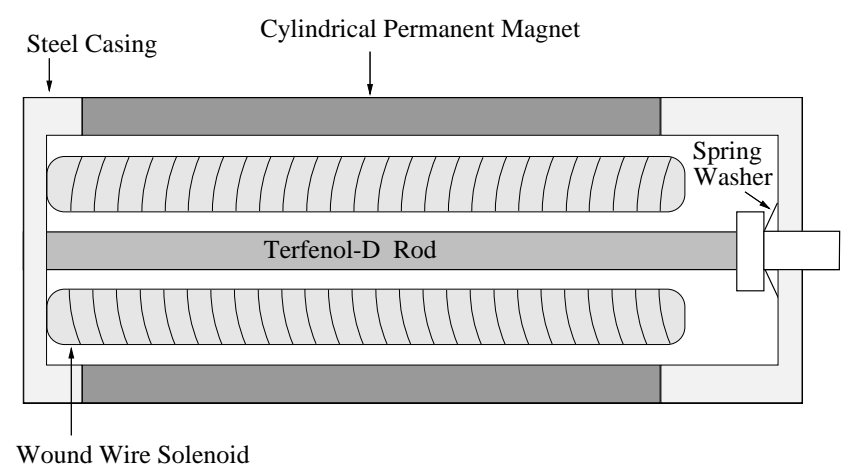

Figure 1. Cross section of a typical Terfenol-D magnetostrictive transducer.

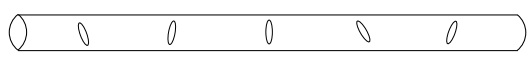

(a)

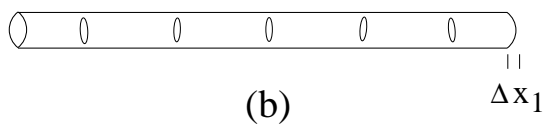

(b)

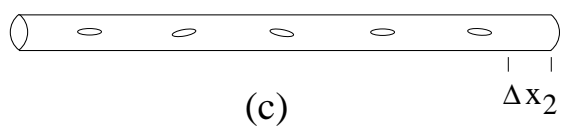

Figure 2. Magnetic moments in the Terfenol-D rod; (a) Orientation of moments in unstressed rod in absence of applied magnetic field; (b) Orientation of moments in prestressed rod with no applied field; (c) Orientation of moments in prestressed rod when field is applied in direction of longitudinal rod axis.

\section{Domain Wall Dynamics}

The model used here is developed through consideration of domain wall theory for ferromagnetic materials. This theory is based upon the observation that below the material's Curie temperature, moments are highly aligned in regions termed domains. The boundaries between domains, in which a transition of the moment orientation occurs, are typically referred to as domain walls. For a material which is free from defects, the domain wall movement is reversible which leads to anhysteretic (hysteresis free) behavior. Most materials, however, contain defects which impede domain wall movement and introduce hysteresis. Details regarding the physics underlying this phenomenon can be found in $[4,5,8]$.

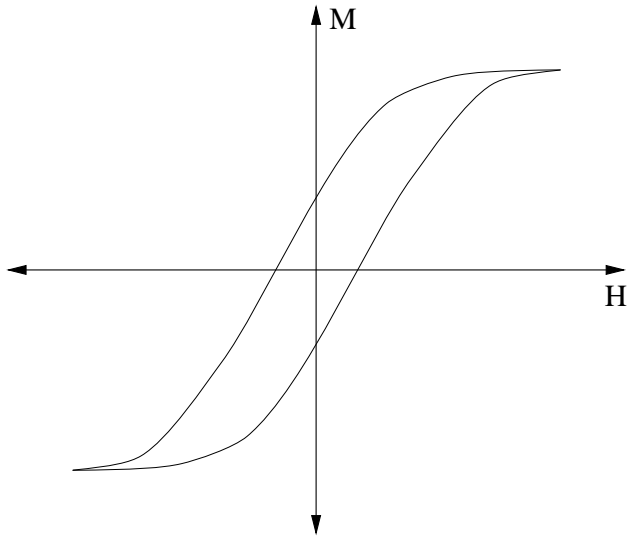

(a)

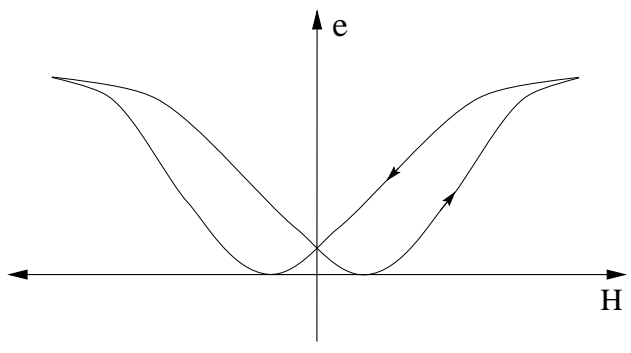

(b)

Figure 3. (a) Relationship between the magnetic field strength $H$ and the magnetization $M$; (b) Applied magnetic field $H$ and resulting strain distribution $e$.

To characterize the magnetization $M$, we consider first the effective field within the material. For rods subjected to a constant prestress $\sigma_{0}$, the effective field is given by

$$
H_{e f f}(t)=H(t)+\alpha M(t)
$$

where

$$
H(t)=n \mathcal{I}(t)
$$

denotes the magnetic field generated by a solenoid having $n$ turns per unit length with an input current $\mathcal{I}(t)$. The parameter $\alpha$ quantifies magnetic and stress interactions. Through thermodynamic considerations, the anhysteretic magnetization is then defined in terms of the Langevin function

$$
M_{a n}(t)=M_{s}\left[\operatorname{coth}\left(\frac{H_{e f f}(t)}{a}\right)-\left(\frac{a}{H_{e f f}(t)}\right)\right] .
$$

Here $M_{s}$ denotes the saturation magnetization of the material and $a$ is a parameter which characterizes the shape of the anhysteretic curve. Energy balancing (see [5]) is then used to quantify the irreversible and reversible magnetizations through the expressions

$$
\frac{d M_{i r r}}{d t}=n \frac{d \mathcal{I}}{d t} \cdot \frac{M_{a n}(t)-M_{i r r}(t)}{k \delta-\alpha\left[M_{a n}(t)-M_{i r r}(t)\right]}
$$


and

$$
M_{r e v}(t)=c\left[M_{a n}(t)-M_{i r r}(t)\right]
$$

( $\delta= \pm 1$ while the constants $c$ and $k$ are estimated from the experimental hysteresis curves). Finally, the total magnetization is given by

$$
M(t)=M_{r \in v}(t)+M_{i r r}(t) .
$$

To first approximation, the strains generated by the material are given by the bulk magnetostriction

$$
\lambda(t)=\frac{3}{2} \frac{\lambda_{s}}{M_{s}^{2}} M^{2}(t)
$$

where $\lambda_{s}$ denotes the saturation magnetostriction (see [4] for details). In combination, (1)-(5) characterize the relationship between the input current $\mathcal{I}$ and the strains generated by the transducer. Details regarding the wellposedness of the model are given in [9].

\section{Structural Model}

To illustrate the use of magnetostrictive actuators in a structural application and provide a setting in which to pose the control problem, we consider a cantilever beam with end-mounted actuators as depicted in Figure 4. As detailed in [2], this setup has been experimentally employed to ascertain properties and capabilities of the actuators.

For modeling purposes, the beam is assumed to have length $\ell$, width $b$, and thickness $h$. The density, Young's modulus, Kelvin-Voigt damping coefficient and air damping coefficient for the beam are denoted by $\rho_{b}, E_{b}, c_{D_{b}}$ and $\gamma$, respectively. The crosssectional area of the Terfenol rod is denoted by $A_{\text {mag }}$ while the Young's modulus and damping coefficient for the Terfenol rod are denoted by $E^{H}$ and $c_{D}^{H}$. The length and width of the connecting bar are denoted by $\ell_{r}$ and $b_{r}$, respectively, while the bar density is given by $\rho_{r}$. Finally, the transverse beam displacement is given by $w$ while $f(t, x)$ denotes an exogenous surface force to the beam.

Moment and force balancing yields the strong form of the Euler-Bernoulli equations

$$
\begin{aligned}
& \rho(x) \frac{\partial^{2} w}{\partial t^{2}}(t, x)+\gamma \frac{\partial w}{\partial t}(t, x)+\frac{\partial^{2} \mathcal{M}_{i n t}}{\partial x^{2}}(t, x) \\
& \quad=f(t, x)+\frac{\partial^{2} \mathcal{M}_{m a g}}{\partial x^{2}}(t, x) \\
& w(t, 0)=\frac{\partial w}{\partial x}(t, 0)=0 \\
& \mathcal{M}_{i n t}(t, \ell)=\frac{\partial \mathcal{M}_{i n t}}{\partial x}(t, \ell)=0
\end{aligned}
$$

along with appropriate initial conditions, as a model for characterizing the transverse beam dynamics. As

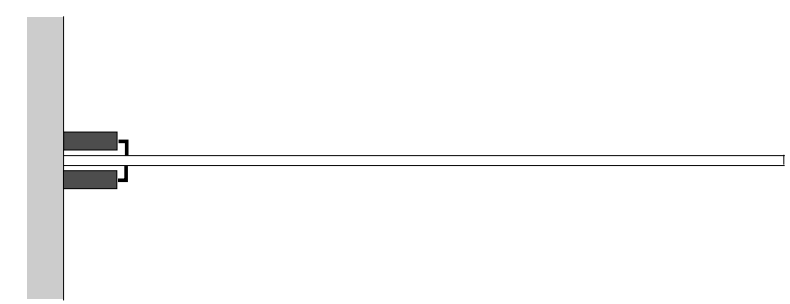

Figure 4. Cantilever beam with magnetostrictive actuators.

detailed in [8], the composite density and internal bending moment are given by

$$
\begin{aligned}
& \rho(x)=\rho_{b} h b+2 \rho_{r} b_{r} \ell_{r} \chi_{\text {rod }}(x) \\
& \mathcal{M}_{\text {int }}(t, x)=E I(x) \frac{\partial^{2} w}{\partial x^{2}}(t, x)+c_{D} I \frac{\partial^{3} w}{\partial x^{2} \partial t}(t, x)
\end{aligned}
$$

where the characteristic function $\chi_{\text {rod }}$ delineates the location of the rods and

$$
\begin{aligned}
& E I(x)=\frac{E_{b} h^{3} b}{12}+2 A_{\text {mag }} E^{H}\left(h / 2+\ell_{r}\right)^{2} \chi_{\text {rod }}(x) \\
& c_{D} I(x)=\frac{c_{D_{b}} h^{3} b}{12}+2 A_{\text {mag }} c_{D}^{H}\left(h / 2+\ell_{r}\right)^{2} \chi_{\text {rod }}(x) .
\end{aligned}
$$

For the case when the Terfenol rods are driven diametrically out-of-phase, the external moment is derived from (5) and is given by

$$
\mathcal{M}_{\text {mag }}(t, x)=\mathcal{K}^{M}\left[M^{2}(t)+2 M(t) M_{s}\right] \chi_{\text {rod }}(x)
$$

where $\mathcal{K}^{M}=\left(3 \lambda_{s} / M_{s}^{2}\right) A_{m a g} E^{H}\left(h / 2+\ell_{r}\right)^{2}$. The inclusion of the weighted magnetization $2 M(t) M_{s}$ provides the bias necessary to attain bidirectional strains.

In order to obtain a weak form of the model, we take the state to be the displacement $w$ in the state space $X=L^{2}(0, \ell)$ with the inner product

$$
\langle\phi, \psi\rangle_{X}=\int_{0}^{\ell} \rho \phi \psi d x
$$

The space of test functions is taken to be $V=$ $H_{L}^{2}(0, \ell) \equiv\left\{\phi \in H^{2}(0, \ell) \mid \phi(0)=\phi^{\prime}(0)=0\right\}$ with the inner product

$$
\langle\phi, \psi\rangle_{V}=\int_{0}^{\ell} E I \phi^{\prime \prime} \psi^{\prime \prime} d x
$$

It should be noted that with these choices, $V$ is continuously and densely embedded in $H$. Hence one has the Gelfand triple

$$
V \hookrightarrow X \simeq X^{*} \hookrightarrow V^{*}
$$

with the pivot space $X$. 
A weak form of the model is then given by

$$
\begin{gathered}
\int_{0}^{\ell} \rho \ddot{w} \psi d x+\int_{0}^{\ell} \gamma \dot{w} \psi d x+\int_{0}^{\ell} \mathcal{M}_{\text {int }} \psi^{\prime \prime} d x \\
=\int_{0}^{\ell} \mathcal{M}_{\text {mag }} \psi^{\prime \prime} d x+\int_{0}^{\ell} f \psi d x
\end{gathered}
$$

for all $\psi \in V$. It is in this form that we develop the approximation method and formulate the control problem.

\section{Approximation Method}

A necessary step for constructing an implementable control law is the approximation of the infinite dimensional system (6). We employ a Galerkin approximation in the spatial variable to obtain a semidiscrete ODE system in time which is amenable to control formulation. Specifically, the spatial basis is taken to be $\left\{B_{j}\right\}_{j=1}^{m+1}$ where $B_{j}(x)$ denotes the $j^{\text {th }}$ cubic $B$-spline modified to satisfy the fixed left boundary condition. Approximate solutions

$$
w^{m}(t, x)=\sum_{j=1}^{m+1} w_{j}(t) B_{j}(x)
$$

are then considered in the subpace $V^{m}=\operatorname{span}\left\{B_{j}\right\}$. To obtain a vector ODE system, the infinite dimensional system (6) is restricted to $V^{m}$ and posed in first-order form to yield

$$
\begin{aligned}
& \dot{y}(t)=A y(t)+[B(u)](t)+F(t) \\
& y(0)=y_{0}
\end{aligned}
$$

The component system matrices have the form

$$
\begin{aligned}
& A=\left[\begin{array}{cc}
0 & I \\
\widetilde{Q}^{-1} K & \widetilde{Q}^{-1} C
\end{array}\right] \\
& {[B(u)](t)=\left[M^{2}(u)+2 M(u) M_{s}\right](t)\left[\begin{array}{c}
0 \\
\widetilde{Q}^{-1} \widetilde{B}
\end{array}\right]} \\
& F(t)=\left[\begin{array}{c}
0 \\
\widetilde{Q}^{-1} \tilde{f}(t)
\end{array}\right]
\end{aligned}
$$

where $y(t)=\left[w_{1}(t), \cdots, w_{m+1}(t), \dot{w}_{1}(t), \cdots, \dot{w}_{m+1}(t)\right]$ and

$$
\begin{array}{ll}
{[\widetilde{Q}]_{i j}=\int_{0}^{\ell} \rho B_{i} B_{j} d x} & {[\widetilde{B}]_{i}=\mathcal{K}^{M} \int_{m a g} B_{i}^{\prime \prime} d x} \\
{[K]_{i j}=\int_{0}^{\ell} E I B_{i}^{\prime \prime} B_{j}^{\prime \prime} d x} & {[\tilde{f}(t)]_{i}=\int_{0}^{\ell} f(t, x) B_{i} d x} \\
{[C]_{i j}=\int_{0}^{\ell} c_{D} I B_{i}^{\prime \prime} B_{j}^{\prime \prime} d x}
\end{array}
$$

Note that $u(t)=\mathcal{I}(t)$ denotes the control input to the system. The system (7) provides the constraints employed in the control problem.

\section{Control Problem}

We consider here the problem of controlling the nonlinear system

$$
\begin{aligned}
& \dot{y}(t)=A y(t)+[B(u)](t) \\
& y\left(t_{0}\right)=y_{0}
\end{aligned}
$$

on the time interval $\left[t_{0}, t_{f}\right]$. As detailed in $[6,7]$, an appropriate performance index for this case is

$$
J(u)=\int_{t_{0}}^{t_{f}} L(y(t), u(t), t) d t+\frac{1}{2} y^{T}\left(t_{f}\right) G y\left(t_{f}\right)
$$

where the Lagrangian is given by

$$
L(y(t), u(t), t)=\frac{1}{2}\left[y^{T}(t) Q y(t)+R u^{2}(t)\right] .
$$

The positive definite matrix $Q$ and positive constant $R$ weight the state and control input, respectively, while the nonnegative matrix $G$ penalizes large terminal values of the state. In the examples which follow, $Q$ and $G$ were chosen to be multiples of the mass matrix and identity, respectively. Finally, the Hamiltonian associated with this system is

$$
H(y, \lambda, u, t)=L(y, u, t)+\lambda^{T}[A y(t)+[B(u)](t)]
$$

where $\lambda \in \mathbb{R}^{m+1}$ is the adjoint variable or Lagrange multiplier. It should be noted that the state equation (8) satisfies

$$
\dot{y}=\frac{\partial H}{\partial \lambda} .
$$

Enforcement of the necessary conditions for minimizing (9) yields the adjoint system

$$
\begin{aligned}
& \dot{\lambda}=-\frac{\partial H}{\partial y} \\
& \lambda\left(t_{f}\right)=-G y\left(t_{f}\right)
\end{aligned}
$$

and the stationary condition

$$
\frac{\partial H}{\partial u}=0
$$

Note that the terminal condition on the adjoint variable is chosen to satisfy the transversality constraint for the system. When combined with the state constraints, this yields the optimality system

$$
\left[\begin{array}{l}
y(t) \\
\lambda(t)
\end{array}\right]=\left[\begin{array}{cl}
A y(t)+[B(u)](t) \\
-A^{T} \lambda(t)+Q y(t)
\end{array}\right], \begin{aligned}
& y\left(t_{0}\right)=y_{0} \\
& \lambda\left(t_{f}\right)=-G y\left(t_{f}\right)
\end{aligned}
$$


where the optimal control satisfies

$$
u^{*}(t)=-R^{-1}\left[B_{u}^{T}\left(u^{*}\right)\right](t) \lambda(t) .
$$

Equivalently, this two point boundary value problem can be expressed as

$$
\begin{aligned}
& \dot{z}(t)=F(t, z) \\
& B_{0} z\left(t_{0}\right)=y_{0} \\
& B_{T} z\left(t_{f}\right)=-G y\left(t_{f}\right)
\end{aligned}
$$

where $z=[y, \lambda]^{T}$ and

$$
\begin{aligned}
& F(t, z)=\left[\begin{array}{c}
A y(t)+[B(u)](t) \\
-A^{T} \lambda(t)+Q y(t)
\end{array}\right] \\
& B_{0}=\left[\begin{array}{ll}
I & 0 \\
0 & 0
\end{array}\right], \quad B_{f}=\left[\begin{array}{ll}
0 & 0 \\
0 & I
\end{array}\right] .
\end{aligned}
$$

The solutions to the system (10) can be approximated through a variety of methods including finite differences and nonlinear multiple shooting. To illustrate a finite difference approach, we consider a discretization of the time interval $\left[t_{0}, t_{f}\right]$ with a uniform mesh having stepsize $\Delta t$ and points $t_{0}, t_{1}, \cdots, t_{N}=t_{f}$. The approximate values of $z$ at these times are denoted by $z_{0}, \cdots, z_{N}$. A forward difference approximation of the temporal derivative then yields the system

$$
\begin{aligned}
& \frac{1}{\Delta t}\left[z_{j+1}-z_{j}\right]=\frac{1}{2}\left[F\left(t_{j}, z_{j}\right)+F\left(t_{j+1}, z_{j+1}\right)\right] \\
& B_{0} z_{0}=\left[y_{0}, 0\right]^{-T} \\
& B_{f} z_{0}=\left[0,-G y\left(t_{f}\right)\right]^{T}
\end{aligned}
$$

for $j=0, \cdots, N-1$. The determination of a solution vector $z_{h}=\left[z_{0}, \cdots, z_{N}\right]$ to (11) can then be expressed as the problem of finding $z_{h}$ which solves

$$
\mathcal{F}\left(z_{h}\right)=0
$$

where $\mathcal{F}$ is defined through the difference method and boundary conditions. The reader is referred to [1] for details.

A quasi-Newton iteration of the form $z_{h}^{k+1}=z_{h}^{k}+\xi_{h}^{k}$, where $\xi_{h}^{k}$ solves

$$
\mathcal{F}^{\prime}\left(z_{h}^{k}\right) \xi_{h}^{k}=-\mathcal{F}\left(z_{h}^{k}\right)
$$

was used to approximate the solution to the nonlinear system (12). Details regarding the efficient solution of the solution (13) by utilizing an analytic LU decomposition of the Jacobian $\mathcal{F}^{\prime}\left(z_{h}^{k}\right)$ will appear in a future paper. We note that for the example presented here, systems having in excess of 20,000 unknowns were resolved with $3-4$ Newton iterations.

\section{Numerical Example}

To illustrate the performance of the control method, we considered a cantilever beam which was excited by a uniform (in space) force

$$
f(t, x)= \begin{cases}100 \sin (10 \pi t) & , \quad t \leq .45 \\ 0 & , \quad t>.45\end{cases}
$$

for 0.45 seconds and was then allowed to freely decay. Control was provided by a pair of end-mounted actuators as depicted in Figure 4. The system was modeled through the modified Euler-Bernoulli model described previously and the dynamics were approximated by numerically integrating the system (7). The dimensions and physical parameters for the system are summarized in Table 1.

The control inputs were computed using the approximation method (11) for the two point boundary value problem $(10)$ on the time interval $\left[t_{0}, t_{f}\right]=[0.45,2.45]$. To illustrate the attenuation yielded by the open loop optimal control method, the uncontrolled and controlled beam displacements at the point $\bar{x}=3 \ell / 5$ are plotted in Figure 5. The corresponding relationship between the input magnetic field and output magnetization is plotted in Figure 6 . It is noted that the model-based nonlinear control law very adequately incorporates the inherent hysteresis in the transducer and provides complete attenuation within 0.5 seconds of being invoked. Both experiments and numerical simulations have demonstrated that linear feedback laws are inadequate in this regime since they do not quantify the energy losses and time delays due to the hysteresis. This illustrates both the necessity for using a nonlinear control method and the effectiveness of the method considered here.

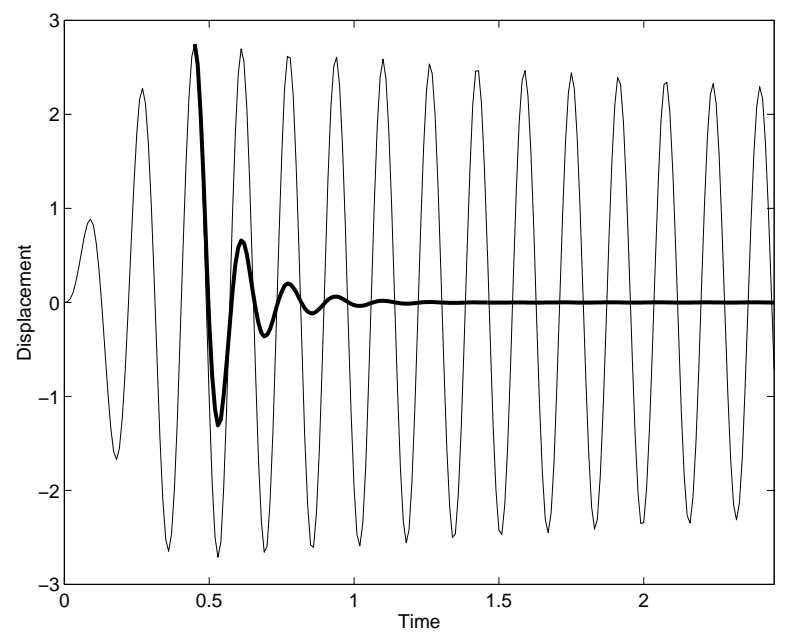

Figure 5. Uncontrolled and controlled beam trajectories at the point $\bar{x}=3 \ell / 5 ;-$ (uncontrolled), (controlled). 


\begin{tabular}{l|l|l}
\hline Beam & Actuator & Terfenol \\
\hline$\ell=.4573 \mathrm{~m}$ & $\ell_{r}=.0254 \mathrm{~m}$ & $a=7105 \mathrm{~A} / \mathrm{m}$ \\
$h=.0016 \mathrm{~m}$ & $b_{r}=.002 \mathrm{~m}$ & $k=7002 \mathrm{~A} / \mathrm{m}$ \\
$b=.0203 \mathrm{~m}$ & $A_{\text {mag }}=.0064 \mathrm{~m}^{2}$ & $\alpha=.007781$ \\
$E_{b}=7.0861 \times 10^{10} \mathrm{~N} / \mathrm{m}^{2}$ & $E^{H}=7.0 \times 10^{10} \mathrm{~N} / \mathrm{m}^{2}$ & $c=0.3931$ \\
$\rho_{b}=2863 \mathrm{~kg} / \mathrm{m}^{3}$ & $\rho_{r}=8524 \mathrm{~kg} / \mathrm{m}^{3}$ & $M_{s}=1.3236 \times 10^{5} \mathrm{~A} / \mathrm{m}$ \\
$c_{D_{b}}=9.3663 \times 10^{5} \mathrm{Ns} / \mathrm{m}^{2}$ & $c_{D}^{H}=0.0$ & $\lambda_{s}=9.96 \times 10^{-4}$ \\
$\gamma=.013 \mathrm{Ns} / \mathrm{m}^{2}$ & & \\
\hline
\end{tabular}

Table 1. Dimensions and parameters for the beam and Terfenol transducer.

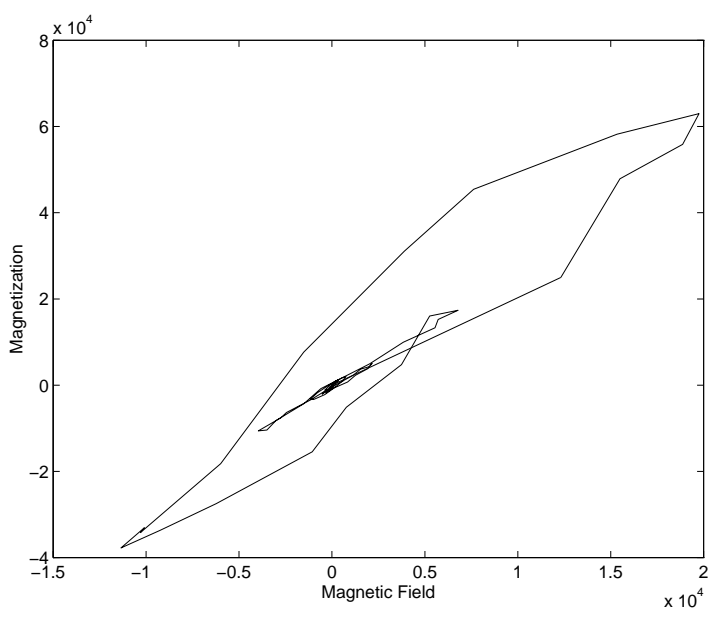

Figure 6. Input magnetic field $H=n \mathcal{I}$ and output magnetization $M$.

\section{Concluding Remarks}

This paper illustrates the development of a modelbased nonlinear control method for magnetostrictive materials. The model is developed through an energy formulation for magnetic domain and domain wall dynamics. This provides a characterization which incorporates the material nonlinearities and hysteresis inherent to the materials. Both experiments and numerical simulations have demonstrated that due to this hysteresis, linear control methods fail at the high drive levels which utilize the full capabilities of the materials. For such regimes, we consider a nonlinear optimal control method which incorporates the hysteresis and nonlinear transducer dynamics. While this method yields an open loop control input which is not robust with regard to perturbations, it provides a means of quantifying the control capabilities of the magnetostrictive materials at high drive levels. It also provides a first step toward the development of robust feedback methods which can be experimentally implemented.

\section{Acknowledgements}

This research was supported in part by the Air Force Office of Scientific Research under grant AFOSR F49620-95-1-0236.

\section{References}

[1] U.M. Ascher, R.M.M. Mattheij and R.D. Russell, Numerical Solution of Boundary Value Problems for Ordinary Differential Equations, SIAM Classics in Applied Mathematics, 1995.

[2] F.T. Calkins, R.L. Zrostlik and A.B. Flatau, "Terfenol-D Vibration Control of a Rotating Shaft," Proc. of the 1994 ASME International Mechanical Engineering Congress and Exposition, Chicago IL; In Adaptive Structures and Composite Materials Analysis and Applications AD-Vol. 45, 1996, pp. 267-274.

[3] D.L. Hall and A.B. Flatau, "Nonlinearities, Harmonics and Trends in Dynamic Applications of Terfenol-D," Proceedings of the SPIE Conference on Smart Structures and Intelligent Materials, Vol. 1917, Part 2, 1993, pp. 929-939.

[4] D.C. Jiles, Introduction to Magnetism and Magnetic Materials, Chapman and Hall, New York, 1991.

[5] D.C. Jiles and D.L. Atherton, "Theory of Ferromagnetic Hysteresis," Journal of Magnetism and Magnetic Materials, 61, 1986, pp. 48-60.

[6] F.L. Lewis and V.L. Syrmos, Optimal Control, John Wiley and Sons, New York, 1995.

[7] E.R. Pinch, Optimal Control and the Calculus of Variations, Oxford University Press, Oxford, 1993.

[8] R.C. Smith, "Modeling Techniques for Magnetostrictive Actuators," CRSC Technical Report CRSCTR97-6; Proceedings of the SPIE, Smart Structures and Integrated Systems, San Diego, CA, March 1997, Vol. 3041, pp. 243-253.

[9] R.C. Smith, "Well-Posedness Issues Concerning a Magnetostrictive Actuator Model," Proceedings of the Conference on Control and Partial Differential Equations, CIRM, Marseille-Luminy, France, June 1997, to appear. 